\title{
OR-1
}

\section{Effects of Andrographolide on Atherosclerosis Induced by Porphyromonas Gingivalis- an Experimental Study in Rat}

\author{
Rami Al Batran ${ }^{1}$, Fouad Al-Bayaty ${ }^{1,}$, Mahmood A. A ${ }^{2}$ and Mazen.M.Jamil ${ }^{1}$ \\ ${ }^{I}$ Center study of periodontology, Faculty of Dentistry, Universiti Teknologi MARA (UiTM), 40450 Shah Alam, Selangor \\ Darul Ehsan, Malaysia; ${ }^{2}$ Department of Molecular Medicine, Faculty of Medicine, University of Malaya, 50603,Kuala \\ Lumpur, Malaysia; E-mail:fouad@salam.uitm.edu.my
}

\begin{abstract}
Associations between atherosclerosis and Porphyromonas gingivalis, a major periodontopathogen, have been indicated. The aim of the present study was to examine the histological and antioxidant effects of Andrographolide on induced atherosclerosis by Porphyromonas gingivalis. Atherogenesis was examined in male Sprague Dawley (SD) rat, which were divided into five groups six rats for each. Normal control (A) and experimental groups (B, C, D and E) were challenged orally with $P$. gingivalis ATCC $33277\left(0.2 \mathrm{~mL}\right.$ of $1.5 \times 10^{\mathrm{x} 12}$ bacterial cells $/ \mathrm{mL}$ in $2 \%$ CMC with PBS) five times a week for one month. Group (C) received orally treatment with simvastatin $100 \mathrm{mg} / \mathrm{kg}$, while groups (D and E) received orally treatment with Andrographolide 20 $\mathrm{mg} / \mathrm{kg}$ and $10 \mathrm{mg} / \mathrm{kg}$ respectively for one month. Animals scarified and sections of the aortic, heart, liver and kidney were made at a thickness of $5 \mu \mathrm{l}$ stained with $(\mathrm{H} \& \mathrm{E})$, and assessed for histopathological changes. Homogenate prepared from the heart and aortic to evaluate the enzymatic activity of SOD and GSH. Histological examination (H\&E) of group (B) aorta sections showed a miner thickening in intimal of the aorta, while treated groups with Andrographolide (D and E) showed no changes in intimal of the aorta. On other hand, histological evaluation of liver, kidney and heart tissues showed no damage or abnormality among groups. Andrographolide significantly improved the enzymatic activity of SOD and GSH in heart and aortic tissue homogenate of group ( $\mathrm{D}$ and E) and that could be due to the anti-oxidants properties of Andrographolide. Conclusion: Based on these results we can say that Andrographolide could be a good herbal agent in the treatment of atherosclerosis.
\end{abstract}

Keywords: Atherosclerosis, Porphyromonas gingivalis, Andrographolide, antioxidant, histology. 